Research Article

\title{
Research on a LADRC Strategy for Trajectory Tracking Control of Delta High-Speed Parallel Robots
}

\author{
Cheng Liu, ${ }^{1}$ Yanming Cheng $\mathbb{D}^{1},{ }^{1}$ Dejun Liu, ${ }^{1}$ Guohua Cao, ${ }^{2}$ and Ilkyoo Lee ${ }^{3}$ \\ ${ }^{1}$ College of Electrical and Information Engineering, Beihua University, Jilin, China \\ ${ }^{2}$ College of Mechanical and Electric Engineering, Changchun University of Science and Technology, Changchun, China \\ ${ }^{3}$ Division of Electrical, Electronics \& Control Engineering, Kongju National University, Cheonan, Republic of Korea
}

Correspondence should be addressed to Yanming Cheng; mycheng@kongju.ac.kr

Received 19 June 2020; Revised 9 August 2020; Accepted 17 August 2020; Published 30 August 2020

Academic Editor: Kalyana C. Veluvolu

Copyright (C) 2020 Cheng Liu et al. This is an open access article distributed under the Creative Commons Attribution License, which permits unrestricted use, distribution, and reproduction in any medium, provided the original work is properly cited.

In order to better track the planned trajectory of Delta high-speed parallel robot, this paper proposes a dynamics control strategy for Delta high-speed parallel robots based on the linear active disturbance rejection control (LADRC) strategy which realizes decoupling control through observing and compensating the coupling and internal and external disturbances between the three joints. Firstly, the structure and dynamics model of the Delta high-speed parallel robot are analyzed, respectively. Secondly, the control scheme of the Delta high-speed parallel robot dynamic LADRC strategy is constructed, and then, the system stability is analyzed. Taking a representative 8-shaped space helical variance trajectory as a given input of the system and a triangular wave as an external disturbance as given disturbance input of the system, simulations are carried out to demonstrate the effectiveness of the proposed LADRC strategy; results indicate that the system with the LADRC strategy has a good quick and precise real-time trajectory tracking and strong robustness.

\section{Introduction}

The parallel robot has the advantages of high speed, lightweight, and strong flexibility, which is complementary to the serial robot. Thereby, an increasing number of researches were carried out by numerous research institutions at home and abroad $[1,2]$. At present, the parallel robot has not been widely used inasmuch as it has a difficult design of the parallel mechanism, difficult kinematics solution, complex trajectory planning, difficult trajectory tracking control, and other problems [2-6]. In this paper, Delta parallel robot is chosen as a study object; the control of Delta parallel robot can be divided into two types: one is kinematics control, which ignores the centripetal force, Coriolis force, and all kinds of disturbances of the robot. It directly controls the rotation angle of the servo motor through the given rotation angle of trajectory planning, which is mainly used in the lowspeed parallel robot; the other is dynamics control. Under the condition of high-speed motion, if the centripetal force, Coriolis force, and all kinds of disturbances are ignored, the operation accuracy of the robot will decrease, and the joints of the robot will vibrate. Therefore, the dynamics control method is designed to improve the dynamic response of the robot system and effectively control all kinds of forces and disturbances in high-speed motion, which realize that the control of the robot in high-speed motion accuracy is of great importance. The controls of three joints of Delta parallel robot are coupled with each other, and the control object has nonlinear characteristics. Therefore, its control has always been a difficult issue in the field of parallel robots; the quality of control strategy directly affects the quality of trajectory tracking, the speed, and operation accuracy of the parallel robot.

The existing control strategies that have been applied to the parallel robot include PID control [7-11], calculated torque control [12-17], and sliding mode variable structure control [18-20]. These methods have higher requirements on the model of the control object, and the operating conditions and operating environment are deterministic. In order to improve the control performance, some scholars 
combined the intelligent control strategy with the traditional control strategy on control of the Delta parallel robot [21-28].

It is generally accepted that classical PID control has good robustness and reliability, and it is easy to be realized. It plays an important role in single input and single output applications. The outputs of three joints of Delta parallel robot are coupled with each other, and the control is nonlinear. The classical PID control is difficult to ensure high-precision and high-speed trajectory tracking and has poor robustness. Therefore, scholars at home and abroad are committed to researching the improved PID control method.

In literature [7-11], starting from the robot joint or joint drive servo system, PD control, PD + speed feed-forward, $\mathrm{PD}+$ position feed-forward, a nonlinear combination of PID parameters, online optimization of PID parameters, fuzzy self-tuning PID, and other controllers as well as various dynamic compensation controllers are used to enable the robot joints to track the given trajectory curve. The method of calculating torque control is to simplify the dynamic model of each joint and carry out decoupling control, which can achieve linear control of each joint. In literature [12-17], the dynamics control of the robot is realized based on the computational torque control method which achieves a good trajectory tracking control. In order to improve the tracking performance of the system, various improvements and compensations are made to predict or calculate the torque in the literature, and the robustness of the system is analyzed. In the nonlinear control system, the sliding mode variable structure control strategy is very popular with researchers, but the controller itself has the problems of chattering and easy to be disturbed. In literature [18-20], a new adaptive variable structure controller, a new smooth sliding mode control algorithm, and a combination of REF neural network and sliding mode control algorithm are used to solve the chattering problem of the traditional sliding mode control, with strong robustness. Since the advent of artificial intelligence control technology in the 1960s, an increasing number of control systems have been added to the elements of artificial intelligence. There are many active figures of artificial intelligence control technology in control of robot trajectory tracking of the robot. Experts and scholars have applied various neural networks, predictive control, fuzzy algorithm, expert system, and other technologies to control of robot trajectory tracking of the robot, which achieve good results. Moreover, the performance of the controller is improved by combining the artificial intelligence method with PID control, calculation torque method, and sliding mode variable structure control, such as the achievements in literature [11, 20, 27-29]. However, the aforementioned methods excessively depend on the model and are difficult to be realized due to the complexity of calculation. Consequently, these methods cannot meet the requirements of high-precision and high-speed control of high-speed parallel robot.

In literature [30], the method of combining the Active Disturbance Rejection Controller (ADRC) with Generalized Proportional Integral (GPI) was adopted. ADRC has the advantage of being model independent, and Generalized Proportional Integral Observer (GPIO) was used instead of Extended State Observer (ESO), which has a better performance comparing with the calculated torque. However, the entire system has many parameters to be tuned, which can achieve good results at a specific load time. Nevertheless, the performance of the system decreases when the load changes, and the parameters cannot be optimized.

In the last few years, Professor Gao Ziqiang proposed the linear active disturbance rejection control method [31] (LADRC) which mainly deals with the linearization of the Extended State Observer and the error feedback combined controller, which is convenient for stability analysis and parameter tuning by using the frequency domain method. The LADRC has been widely used in a wider range owing to its advantages of simple parameter tuning and strong antiinterference ability, and more achievements have been achieved in some literature [32-38]. Motivated by the aforementioned analysis, this paper proposes an LADRC strategy to apply to the dynamic stability control of Delta high-speed parallel robot for the first time. Simulation results demonstrate that the system with LADRC strategy has the performance of a good trajectory tracking and strong robustness.

\section{Structure and Dynamics Modeling of Delta High-Speed Parallel Robots}

2.1. Structure of Delta High-Speed Parallel Robots. In this paper, the structure sketch of Delta high-speed parallel robot is depicted in Figure 1. A1A2A3 is a fixed platform, B1B2B3 is a mobile platform, $\mathrm{Ai}$ is a rotating joint, $\mathrm{Bi}$ and $\mathrm{Ci}$ are spherical joints, $\mathrm{AiCi}$ is an active arm, and $\mathrm{CiBi}$ is a driven arm, $i=1,2,3$. $O$ is the center of the fixed platform, and $P$ is the center of the mobile platform. $R$ is the outer circle radius of the fixed platform, and $r$ is the outer circle radius of the mobile platform. Both $\mathrm{A} 1 \mathrm{~A} 2 \mathrm{~A} 3$ and $\mathrm{B} 1 \mathrm{~B} 2 \mathrm{~B} 3$ are regular triangles.

2.2. Dynamics Modeling of Delta High-Speed Parallel Robots. According to the literature [30], the dynamics equation of delta high-speed parallel robot can be expressed by

$$
M(\theta) \ddot{\theta}+C(\theta, \dot{\theta}) \dot{\theta}+G(\theta)=\tau .
$$

Add friction force and disturbance to formula (1), and formula (2) can be obtained as

$$
M(\theta) \ddot{\theta}+C(\theta, \dot{\theta}) \ddot{\theta}+G(\theta)+F(\dot{\theta})+\tau_{d}=\tau,
$$

where $\theta \in R^{n}$ is the rotation angle of the three joints of Delta high-speed parallel robot; $M(\theta) \in R^{n x n}$ is inertia matrix of the three joints of Delta high-speed parallel robot; $C(\theta, \dot{\theta})$ is centripetal force and Coriolis force; $G(\theta) \in R^{n}$ is the gravity term; $F(\dot{\theta}) \in R^{n}$ is friction; $\tau_{d}$ is disturbance term; and $\tau$ is servo input.

Formula (2) is transformed into formula (3), as 


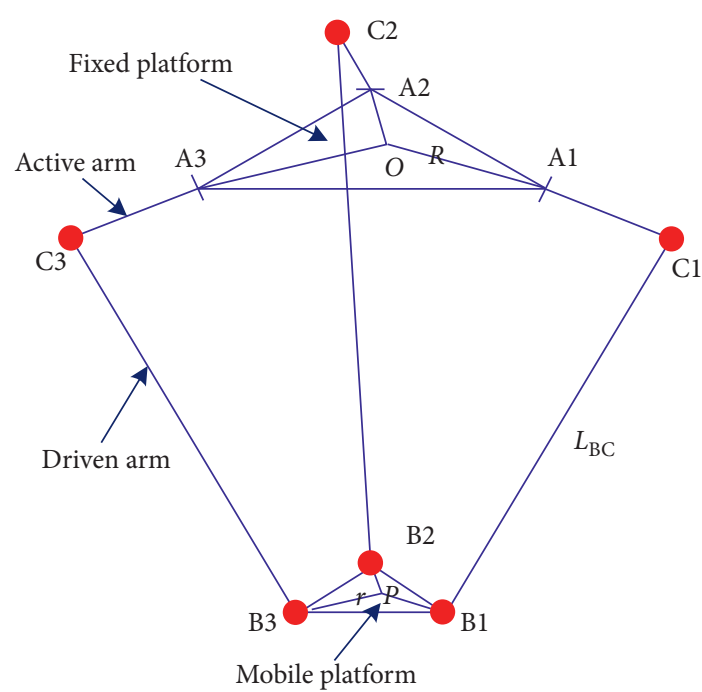

Figure 1: Mechanism sketch of three-degree-of-freedom Delta high-speed parallel robots.

$$
\ddot{\theta}=-M^{-1} C(\theta, \dot{\theta}) \dot{\theta}-M^{-1} G(\theta)-M^{-1} F(\dot{\theta})-M^{-1}(\theta) \tau_{d}+M^{-1} \tau .
$$

\section{Study on LADRC Strategy}

3.1. Scheme of $L A D R C$. The mechanical structure of the Delta high-speed parallel robot is complex, and the three joints are coupled with each other. The control structure is shown in Figure 2. $X_{1}(s), X_{2}(s)$, and $X_{3}(s)$ are inputs of Delta high-speed parallel robot. $Y_{1}(s), Y_{2}(s)$, and $Y_{3}(s)$ are outputs of the Delta high-speed parallel robot. When one $X_{i}(s)$ is changed, at the same time, the other two $X_{i}(s)$ will follow it, and three $Y_{i}(s)$ will change. Thereby, in order to realize the trajectory tracking control of Delta high-speed parallel robot, three joints need to be input at the same time and decoupled each other.

When the parallel robot is running, the decomposed load on each joint changes nonlinearly with the change of pose and angular acceleration, which cannot be expressed linearly. Therefore, PID control cannot meet the requirements of the high-speed and high-precision control system of Delta high-speed parallel robot. According to the motion requirements and nonlinear characteristics of Delta highspeed parallel robot, this paper adopts the LADRC strategy to control Delta high-speed parallel robot. An 8-shaped space helical variance trajectory as the input signal is used to verify the trajectory tracking of the control strategy.

Figure 3 shows the LADRC strategy. The LADRC strategy has strong robustness, in that many uncertain factors are omitted in dynamics modeling. In practical control, these are uncertain factors of control, while the LADRC strategy does not depend on the establishment of the dynamics model, which circumvents the error problem of the dynamics model of the parallel robot. Control idea is to control each joint separately; the coupling parameters between the joints, the omitted factors in modeling, and the disturbance in actual operation are all treated as the disturbance to compensate.

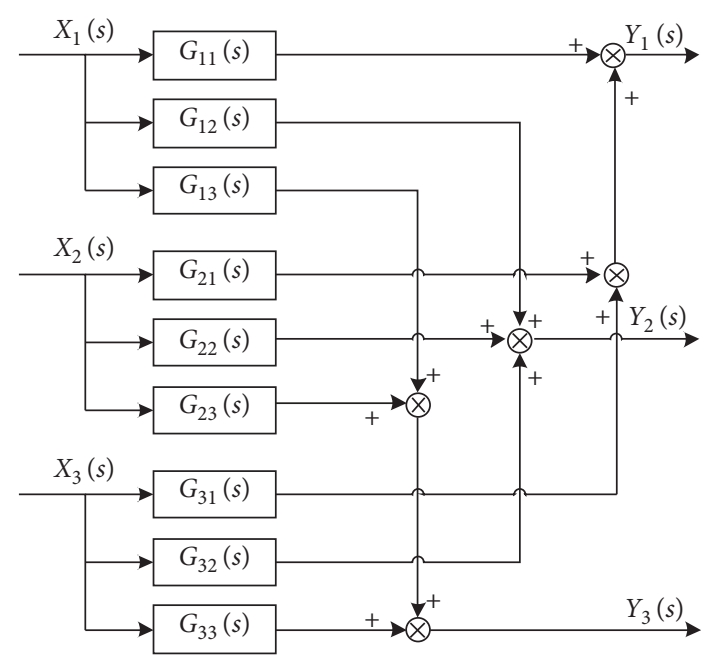

Figure 2: Control system structure of Delta high-speed parallel robot.

Finally, the high-precision trajectory tracking control of Delta high-speed parallel robot is realized.

3.2. Design of System Controller. The dynamics of Delta highspeed parallel robot are expressed by formula (3) which can be converted into

$$
\ddot{\theta}=-a_{1} \dot{\theta}-a_{2} \theta+f_{0}(\theta, \dot{\theta}, \omega, t)+b \mu,
$$

where $\mu$ is the input of the system; $\theta$ is the output of the system; $-a_{1} \dot{\theta}-a_{2} \theta$ is known modeling dynamics of object; $f_{0}(\theta, \dot{\theta}, \omega, t)$ is unknown modeling dynamics of objects and sum of external disturbances; $b$ is the uncertain control gain; and $b_{0}$ is the approximate value of uncertain control gain $b$; let $f(\theta, \dot{\theta}, \omega, t)=f(\cdot)-a_{1} \dot{\theta}-a_{2} \theta+f_{0}(\theta, \dot{\theta}, \omega, t)+\left(b-b_{0}\right) \mu$ which is called "total disturbance."

Let $x_{1}=\theta, x_{2}=\dot{\theta}$, and $x_{3}=f(\cdot)$. Formula (4) expresses state equation of the second-order objects which can be written as

$$
\begin{aligned}
& \dot{x}_{1}=x_{2}, \\
& \dot{x}_{2}=x_{3}+b_{0} \mu, \\
& \dot{x}_{3}=h+\dot{f} .
\end{aligned}
$$

The state equation of the LESO (Linear Extended State Observer) designed for formula (3) is expressed as follows:

$$
\begin{aligned}
e & =z_{1}-\theta, \\
\dot{z}_{1} & =z_{2}-\beta_{1} f_{1}(e), \\
\dot{z}_{2} & =z_{3}-\beta_{2} f_{2}(e)+b_{0} \mu, \\
\dot{z}_{3} & =-\beta_{3} f_{3}(e) \\
\mu & =\frac{-z_{3}+\mu_{0}}{b_{0}}
\end{aligned}
$$

where $L$ is the gain of LESO; $L=\left[\begin{array}{lll}\beta_{1} & \beta_{2} & \beta_{3}\end{array}\right]^{\mathrm{T}}$, $f_{i}(e)=z_{1}-\theta(i=1,2,3)$, and $\beta_{1}, \beta_{2}$, and $\beta_{3}$ are selected 


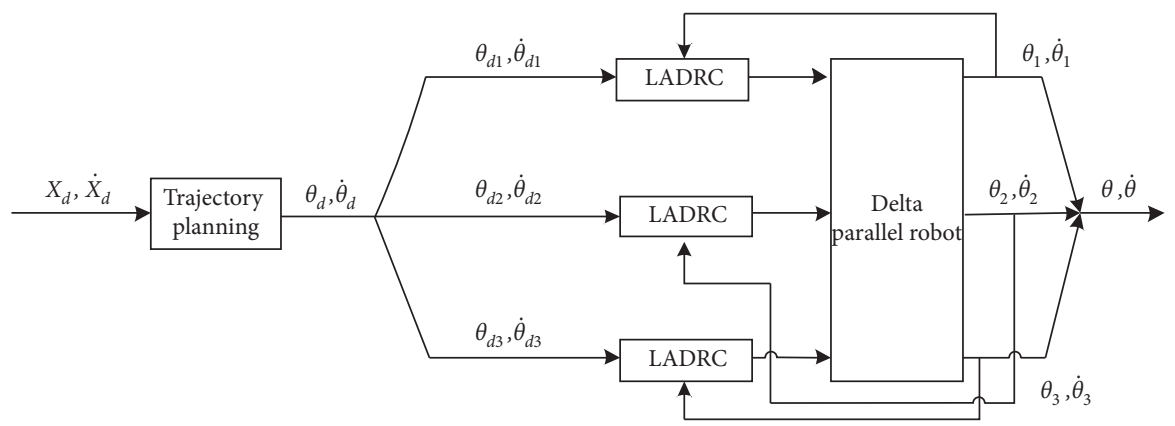

FIGURE 3: Delta high-speed parallel robot based on LADRC.

appropriate gains of LESO, respectively; LESO can track all variables including $z_{1}=\theta, z_{2}=\dot{\theta}$, and $z_{3}=f(\cdot)$ in the system expressed through formula (6) in real time.

Linear control law can be expressed by

$$
u_{0}=k_{p}\left(v-z_{1}\right)+k_{d}\left(\dot{v}-z_{2}\right) \text {. }
$$

According to formula (6),

$$
\begin{aligned}
& z_{1}=\frac{\beta_{1} s^{2}+\beta_{2} s+\beta_{3}}{s^{3}+\beta_{1} s^{2}+\beta_{2} s+\beta_{3}} \theta+\frac{b_{0} s}{s^{3}+\beta_{1} s^{2}+\beta_{2} s+\beta_{3}} \mu, \\
& z_{2}=\frac{\left(\beta_{2} s+\beta_{3}\right) s}{s^{3}+\beta_{1} s^{2}+\beta_{2} s+\beta_{3}} \theta+\frac{b_{0}\left(s+\beta_{1}\right) s}{s^{3}+\beta_{1} s^{2}+\beta_{2} s+\beta_{3}} \mu, \\
& z_{3}=\frac{\beta_{3} s^{2}}{s^{3}+\beta_{1} s^{2}+\beta_{2} s+\beta_{3}} \theta-\frac{b_{0} \beta_{3}}{s^{3}+\beta_{1} s^{2}+\beta_{2} s+\beta_{3}} \mu .
\end{aligned}
$$

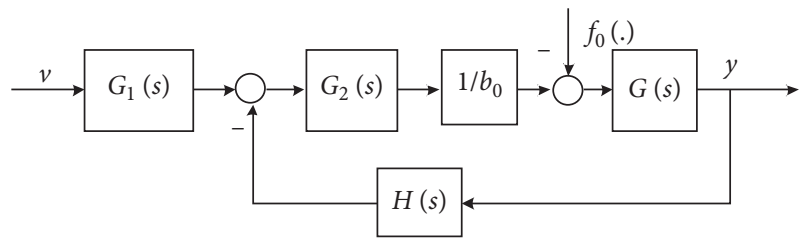

Figure 4: The single closed-loop structure diagram.

According to formula (8),

$$
\mu_{0}=\frac{1}{b_{0}}\left[k_{p}\left(v-z_{1}\right)+k_{d}\left(\dot{v}-z_{2}\right)-z_{3}\right] .
$$

Substitute $z_{1}, z_{2}$, and $z_{3}$ into formula (11) as follows:

$$
\mu=\frac{1}{b_{0}} \frac{s^{3}+\beta_{1} s^{2}+\beta_{2} s+\beta_{3}}{s^{3}+\left(\beta_{1}+k_{d}\right) s^{2}+\left(\beta_{2}+k_{p}+k_{d} \beta_{1}\right) s}\left[\left(k_{p}+k_{d} s\right) v \frac{\left(k_{p} \beta_{1}+k_{d} \beta_{2}+\beta_{3}\right) s^{2}+\left(k_{p} \beta_{2}+k_{d} \beta_{3}\right) s+k_{p} \beta_{3}}{s 3+\beta_{1} s^{2}+\beta_{2} s+\beta_{3}}\right] .
$$

3.2.1. Stability Analysis. From equation (13), the single closed-loop structure diagram is shown in Figure 4.

One has

$$
\begin{aligned}
G_{1}(s) & =k_{p}+k_{d} s \\
G_{2}(s) & =\frac{s^{3}+\beta_{1} s^{2}+\beta_{2} s+\beta_{3}}{s^{3}+\left(\beta_{1}+k_{d}\right) s^{2}+\left(\beta_{2}+k_{p}+k_{d} \beta_{1}\right) s} \\
H(s) & =\frac{\left(k_{p} \beta_{1}+k_{d} \beta_{2}+\beta_{3}\right) s^{2}+\left(k_{p} \beta_{2}+k_{d} \beta_{3}\right) s+k_{p} \beta_{3}}{s^{3}+\beta_{1} s^{2}+\beta_{2} s+\beta_{3}} .
\end{aligned}
$$

From the structure diagram in Figure 4, the closed-loop transfer function can be obtained as

$$
G_{b}(s)=\frac{\left(\left(G_{1}(s) G_{2}(s) G(s)\right) / b_{0}\right)}{1+\left(\left(G_{2}(s) G(s)\right) / b_{0}\right) H(s)}
$$

(1) The Scenario of Precisely Known Controlled Object. It can be seen from the above analysis that dynamics model formula (3) of the parallel robot is established by omitting the moment of inertia and the friction between joints; thereby, the model is considered to be known.

Theorem 1. When the model of the controlled object is known accurately, the differential tracker does not affect the stability of the system but only affects the zero point of the system. Reasonable selection of $b_{0}, k_{p}, k_{d}, \beta_{1}, \beta_{2}$, and $\beta_{3}$ can make the system stable.

Proof. If the object model is known precisely, its transfer function is

$$
G(s)=\frac{k_{0}}{s^{2}+a_{1} s+a_{2}} .
$$

Substituting formulae (14)-(18) into formula (17), one obtains 


$$
G_{b}(s)=\frac{\left(k_{p}+k_{d} s\right)\left[s^{3}+\beta_{1} s^{2}+\beta_{2} s+\beta_{3}\right] k_{0}}{b_{0}\left\{\left[s^{3}+\left(\beta_{1}+k_{d}\right) s^{2}+\left(\beta_{2}+k_{p}+k_{d} \beta_{1}\right) s\right]\left[s^{2}+a_{1} s+a_{2}\right]\right\}+k_{0}\left[\left(k_{p} \beta_{1}+k_{d} \beta_{2}+\beta_{3}\right) s^{2}+\left(k_{p} \beta_{2}+k_{d} \beta_{3}\right) s+k_{p} \beta_{3}\right]} .
$$

Closed-loop characteristic equation is presented as

$$
\begin{aligned}
D(s)= & b_{0} s^{5}+b_{0}\left(\beta_{1}+k_{d}+a_{1}\right) s^{4}+b_{0}\left(\beta_{2}+k_{p}+k_{d} \beta_{1}+a_{1}\left(\beta_{1}+k_{d}\right)+a_{2}\right) s^{3} \\
& +\left[b_{0} a_{1}\left(\beta_{2}+k_{p}+k_{d} \beta_{1}\right)+b_{0} a_{2}\left(\beta_{1}+k_{d}\right)+k_{0}\left(k_{p} \beta_{1}+k_{d} \beta_{2}+\beta_{3}\right)\right] s^{2}+\left[b_{0} a_{2}\left(\beta_{2}+k_{p}+k_{d} \beta_{1}\right)\right. \\
& \left.+k_{0}\left(k_{p} \beta_{2}+k_{d} \beta_{3}\right)\right] s+k_{0} k_{p} \beta_{3} .
\end{aligned}
$$

The following constraint can be specified:

$$
\begin{aligned}
D_{0}= & b_{0}, \\
D_{1}= & b_{0}\left(\beta_{1}+k_{d}+a_{1}\right), \\
D_{2}= & b_{0}\left(\beta_{2}+k_{p}+k_{d} \beta_{1}+a_{1} \beta_{1}+a_{1} k_{d}+a_{2}\right), \\
D_{3}= & {\left[b_{0} a_{1}\left(\beta_{2}+k_{p}+k_{d} \beta_{1}\right)+b_{0} a_{2}\left(\beta_{1}+k_{d}\right)\right.} \\
& \left.+k_{0}\left(k_{p} \beta_{1}+k_{d} \beta_{2}+\beta_{3}\right)\right], \\
D_{4}= & {\left[b_{0} a_{2}\left(\beta_{2}+k_{p}+k_{d} \beta_{1}\right)+k_{0}\left(k_{p} \beta_{2}+k_{d} \beta_{3}\right)\right], } \\
D_{5}= & k_{0} k_{p} \beta_{3} .
\end{aligned}
$$

From Routh criterion, the stability of the system can be obtained as

$$
\begin{array}{rlll}
s^{5} & D_{0} & D_{2} & D_{4}, \\
s^{4} & D_{1} & D_{3} & D_{5}, \\
s^{3} & B_{31} & B_{32}, \\
s^{2} & B_{41} & B_{42}, \\
s^{1} \quad B_{51}, & \\
s^{0} \quad B_{61}, & \\
B_{31} & =\frac{D_{1} D_{2}-D_{0} D_{3}}{D_{1}}, \\
B_{32} & =\frac{D_{1} D_{4}-D_{0} D_{5}}{D_{1}}, \\
B_{41} & =\frac{B_{31} D_{3}-D_{1} B_{32}}{B_{31}}, \\
B_{42} & =D_{5}, \\
B_{51} & =\frac{B_{41} B_{32}-B_{31} D_{5}}{B_{41}}, \\
& =D_{5} .
\end{array}
$$

(2) The Scenario of Unknown Parameters of the Controlled Object. When Delta high-speed parallel robot runs at a high speed, the coupling relationships between joints are significantly relevant to the effects of the moment of inertia and friction between joints. They will result in the system chatter in the process of operation and ultimately destroy the stability of the system and decrease trajectory tracking performance if these impact factors were not taken into account. Therefore, in the design of the LADRC strategy, these factors are taken into consideration as unknown model parameters of the system when carrying out modeling.

The stability of the system model is proved as follows. Let the nominal model of the object be $G_{n}$, and then the actual object is $G(s)=G_{n}(1+\delta G(s))$; $\delta G$ is the perturbation of nominal model and meets $|\delta G(j \omega)| \leq \overline{\delta(G \omega)} ; \overline{\delta G(\omega)}$ is bounded the uncertainty of multiplicative norm.

Owing to the closed-loop characteristic equation $1+G_{2}(s)\left(1 / b_{0}\right) G(s) H(s)=0$, the following expression is obtained as

$$
1+G_{2}(s) \frac{1}{b_{0}} G_{n}(s)(1+\delta G) H(s)=0 .
$$

According to robust stability criterion, for arbitrary $\omega$, the inequality satisfying formula (21) is as follows:

$$
\begin{gathered}
b_{0}+G_{2}(s) G_{n}(s) H(s)+G_{2}(s) G_{n}(s) H(s) \delta G(s)=0, \\
\overline{\delta G(\omega)}<\Delta G(\omega)=\left|\frac{b_{0}+G_{2}(s) G_{n}(s) H(s)}{G_{2}(s) G_{n}(s) H(s)}\right|
\end{gathered}
$$

The conditions given by formula (22) can stabilize the system.

It can be seen that selecting $b_{0}, k_{p}, k_{d}, \beta_{1}, \beta_{2}$, and $\beta_{3}$ properly can make the system stable, which can guarantee that the system has the stability and certain robustness.

It can be concluded from the above stability proof that the LADRC strategy not only realizes the dynamics control of the trajectory tracking of Delta high-speed parallel robot but also guarantees that the uncertain factors of the model will not affect the stability of the system at high-speed operation. 
3.2.2. Design of Delta High-Speed Parallel Robots. The controller structure of the LADRC strategy is shown in Figure 5.

As shown in Figure 5, the LADRC strategy only needs to be set by setting up stability conditions of LESO which are $\beta_{1}>0, \beta_{2}>0$, and $\beta_{3}>0$ and meet $\beta_{1} \beta_{2}>\beta_{3}$.

As depicted in Figure 2, Delta high-speed parallel robot is composed of three inputs $X_{1}(\mathrm{~s}), X_{2}(\mathrm{~s})$, and $X_{3}(\mathrm{~s})$ and three outputs $Y_{1}(\mathrm{~s}), Y_{2}(\mathrm{~s})$, and $Y_{3}(\mathrm{~s})$, which are coupled with each other and can be constructed as shown in the following formula:

$$
\left\{\begin{array}{l}
Y_{1}(\mathrm{~s})=G_{11}(\mathrm{~s}) X_{1}(\mathrm{~s})+G_{12}(\mathrm{~s}) X_{2}(\mathrm{~s})+G_{13}(\mathrm{~s}) X_{3}(\mathrm{~s}) \\
Y_{2}(\mathrm{~s})=G_{21}(\mathrm{~s}) X_{1}(\mathrm{~s})+G_{22}(\mathrm{~s}) X_{2}(\mathrm{~s})+G_{23}(\mathrm{~s}) X_{3}(\mathrm{~s}) \\
Y_{3}(\mathrm{~s})=G_{31}(\mathrm{~s}) X_{1}(\mathrm{~s})+G_{32}(\mathrm{~s}) X_{2}(\mathrm{~s})+G_{33}(\mathrm{~s}) X_{3}(\mathrm{~s})
\end{array}\right.
$$

According to formulas (6) and (23), the system equation of Delta high-speed parallel robot can be obtained as expressed in

$$
\begin{aligned}
& \ddot{\theta}_{1}=f_{1}\left(\theta_{1}, \dot{\theta}_{1}, \mu_{1}(t), t\right)+k_{1} \mu_{1}, \\
& \ddot{\theta}_{2}=f_{2}\left(\theta_{2}, \dot{\theta}_{2}, \mu_{2}(t), t\right)+k_{2} \mu_{2}, \\
& \ddot{\theta}_{3}=f_{1}\left(\theta_{3}, \dot{\theta}_{3}, \mu_{3}(t), t\right)+k_{3} \mu_{3},
\end{aligned}
$$

where $f_{1}\left(\theta_{1}, \dot{\theta}_{1}, \mu_{1}(t), t\right), \quad f_{2}\left(\theta_{2}, \dot{\theta}_{2}, \mu_{2}(t), t\right)$, and $\left(f_{3}\left(\theta_{3}, \dot{\theta}_{3}, \mu_{3}(t), t\right)\right)$ are equivalent comprehensive disturbance which are the comprehensive functions of the coupling term between the three axes, the uncertainty term in high-speed motion, and various disturbance terms in the field. The control structure block diagram is shown in Figure 6.

\section{Simulation and Results Analysis}

In this section, to verify the performance of the controller based on the proposed LADRC strategy, the primary parameters of Delta high-speed parallel robot are summarized in Table 1.

An 8-shaped trajectory that is a representative space helical variance trajectory is taken as inputs of the system, which is expressed as

$$
\left\{\begin{array}{l}
x_{1}=t \\
x_{2}=800 \sin \left(\left(0.3 t+\frac{1}{360}\right) * \pi\right), \\
x_{3}=800 \sin (0.6 t * \pi) .
\end{array}\right.
$$

The pictures of different views corresponding to formula (25) are depicted in Figure 7.

For obtaining trajectories under some conditions, the first step is to verify the performance of the system when the disturbance is not introduced and then verify the performance of the system when the disturbance is introduced. To better estimate, the control effect, the angle

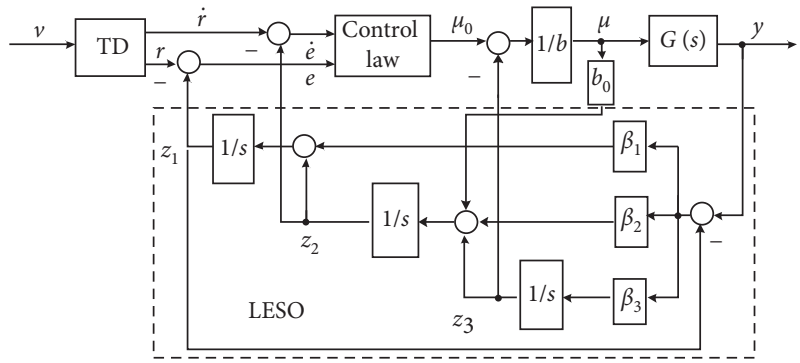

FIGURE 5: Structure diagram of LADRC for the second-order system.

limit, time (speed) limit, and space limit of the robot are removed.

Case 1. The case of 8-shaped trajectory input without disturbance.

According to $z_{i}(i=1,2,3)$ in formula $\mathrm{fd} 9(9)$, stability conditions of LESO must meet $\beta_{1}>0, \beta_{2}>0, \beta_{3}>0$, and $\beta_{1} \beta_{2}>\beta_{3}$. On this basis, it is concluded that coefficients $\beta_{1}=1, \beta_{2}=65000$, and $\beta_{3}=600$ through a large number of simulation experiments. The trajectory of Delta high-speed parallel robot is set as the 8-shaped trajectory. The given trajectories of joint 1 , joint 2 , and joint 3 are set according to formula (25). According to Figure 6, the simulation curve of trajectory tracking control is obtained in Figure 8, the blue circle denotes the given circular trajectory, and the red curve denotes the trajectory controlled by the LADRC strategy.

In order to verify the robustness of the controller against disturbance, a triangular wave external disturbance with an amplitude of $50 \mathrm{~mm}$ and a period of $0.2 \mathrm{~Hz}$ is added after the Delta high-speed parallel robot runs to $5 \mathrm{~s}$. The disturbance signal is shown in Figure 9.

Figure 10 shows the tracking trajectories of three joints in the scenario of only PID control without the LADRC strategy.

The tracking trajectory of only using PID control under the condition of without the LADRC strategy is obtained in Figure 11.

From Figures 10 and 11, it is very obvious that only using PID control completely cannot control the tracking trajectory. Therefore, it is very necessary to introduce the LADRC strategy to resolve the antidisturbance of PID control. Figures 12 and 13 show the effects of tracking trajectory control when the LADRC strategy is used.

In Figure 13, the fluctuation of the three-dimensional trajectory is not obvious, and the 8-shaped trajectory is slightly vibrated. It is verified that the LADRC strategy can realize the decoupling control of three joints and has strong robustness under the disturbance of the triangular wave for a representative space helical variance trajectory of 8-shaped trajectory input.

Simulation results show that the LADRC strategy can realize the decoupling control of three joints and realize the quick and precise real-time tracking of the given trajectory and has good robustness. 


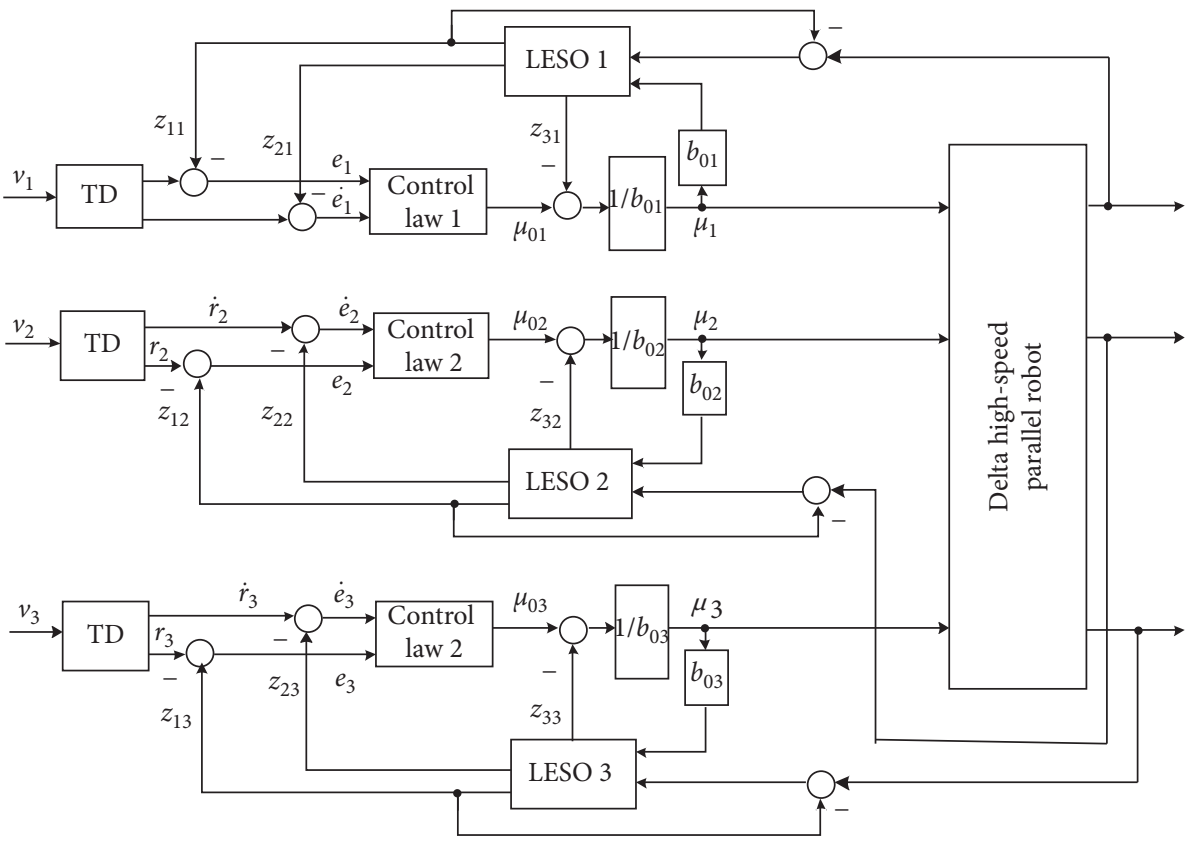

FigURE 6: Simulation structure block diagram.

TABle 1: Primary parameters of Delta high-speed parallel robot for simulation.

\begin{tabular}{lc}
\hline Parameters & Value \\
\hline Rated output power of servo motor & $750 \mathrm{~W}$ \\
Rated speed of servo motor & $3000 \mathrm{rpm}$ \\
Rated torque of servo motor & $2.38 \mathrm{M} \cdot \mathrm{m}$ \\
Moment of inertia of servo motor & $1.59 \times 10^{-4}\left(\mathrm{~kg} / \mathrm{m}^{2}\right)$ \\
Reduction ratio & $20: 1$ \\
Mass of active arm & $2.35 \mathrm{~kg}$ \\
Mass of driven arm & $0.9 \mathrm{~kg}$ \\
Length of active arm & $400 \mathrm{~mm}$ \\
Length of driven arm & $1000 \mathrm{~mm}$ \\
\hline
\end{tabular}

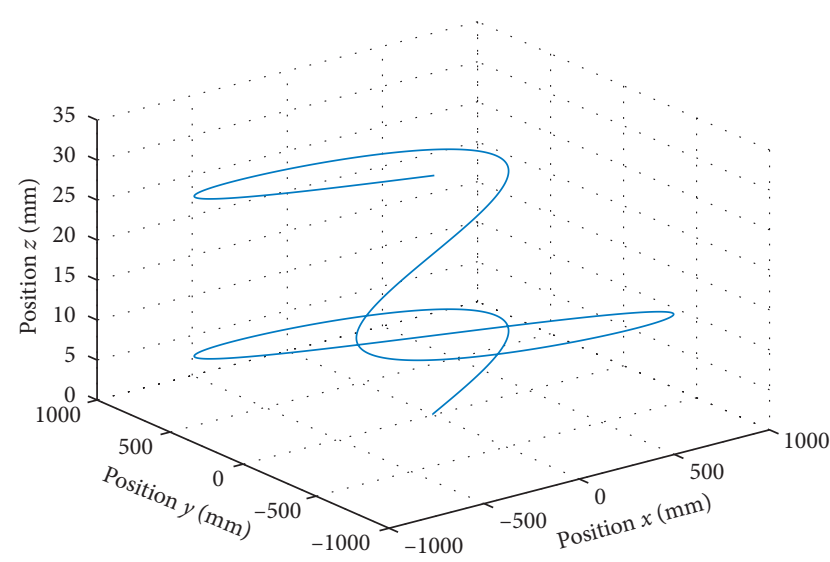

(a)

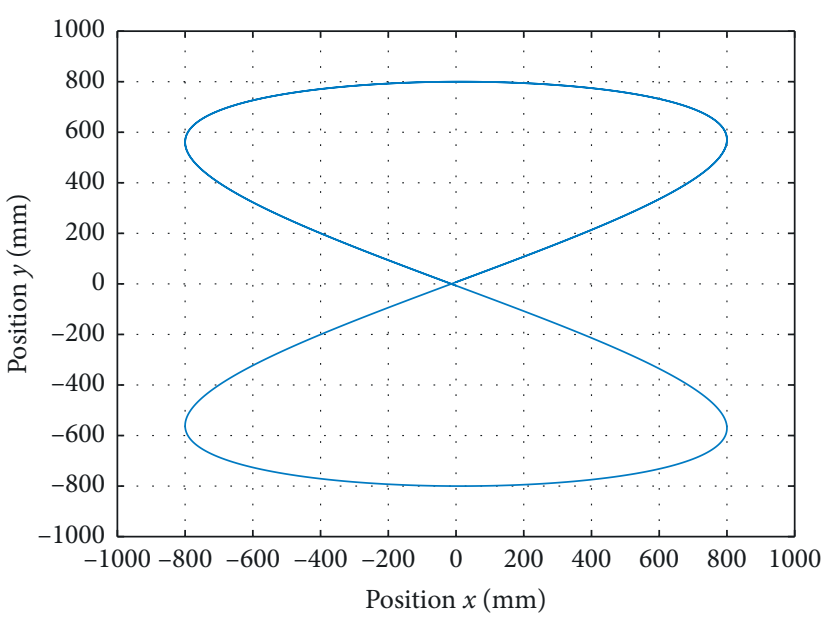

(b)

Figure 7: Continued. 


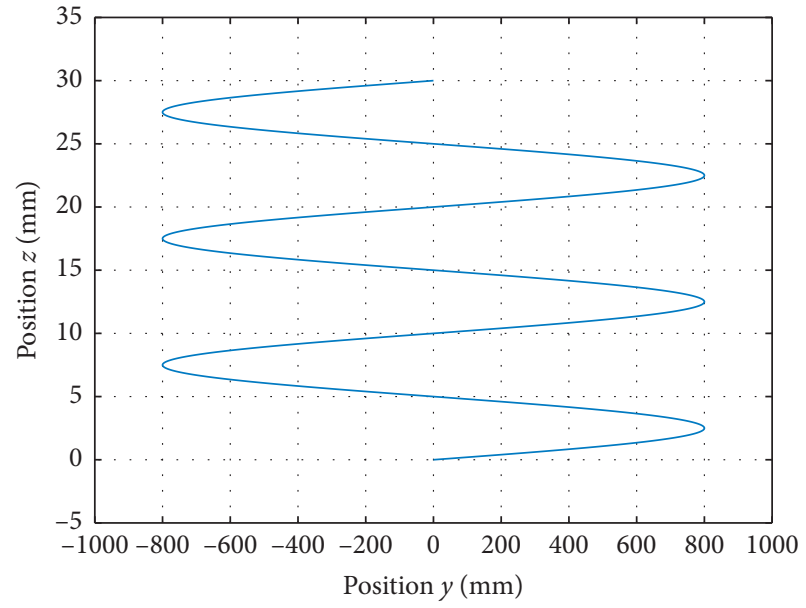

(c)

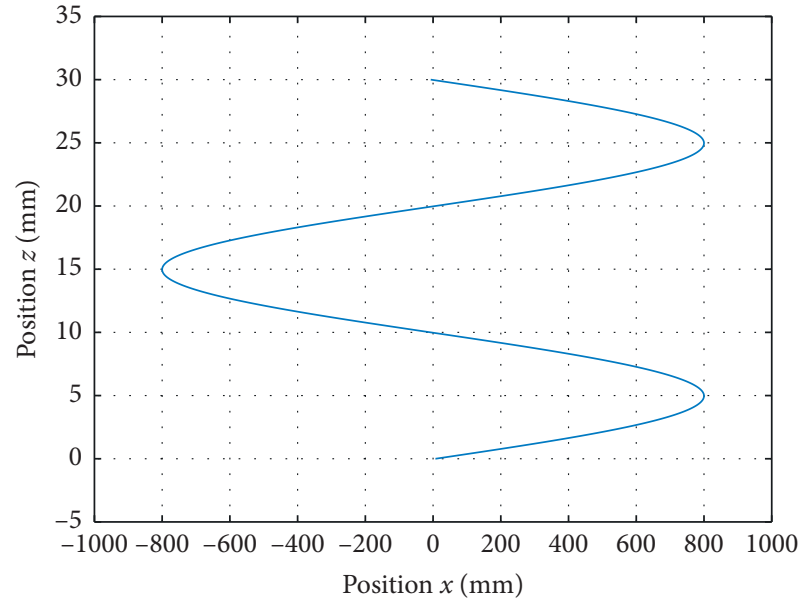

(d)

Figure 7: 8-shaped trajectory. (a) The space trajectory in 3D coordination system. (b) The projection on the $x-y$ plane. (c) The projection on the $y-z$ plane. (d) The projection on the $x-z$ plane.

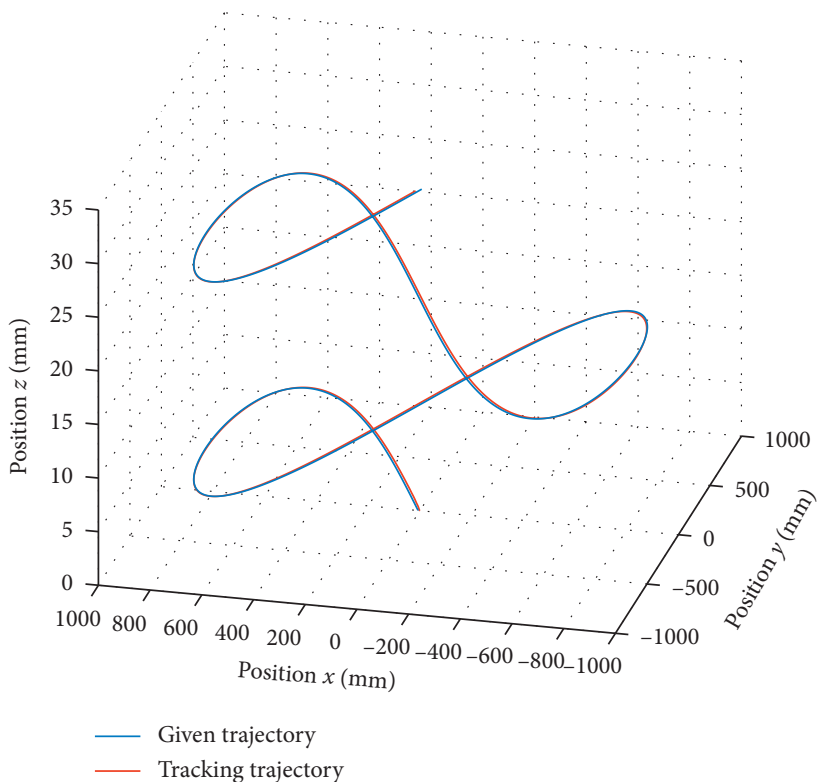

Figure 8: Trajectories without disturbance.

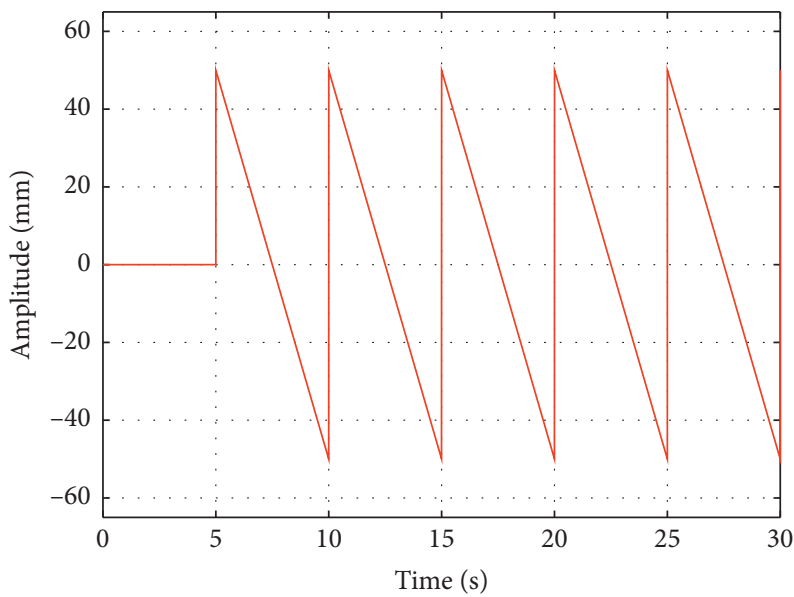

FIgURe 9: Disturbance signal. 


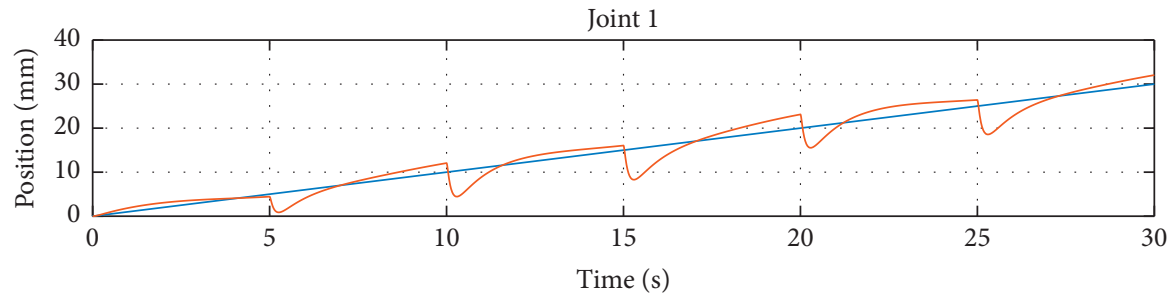

Given trajectory

Tracking trajectory

(a)

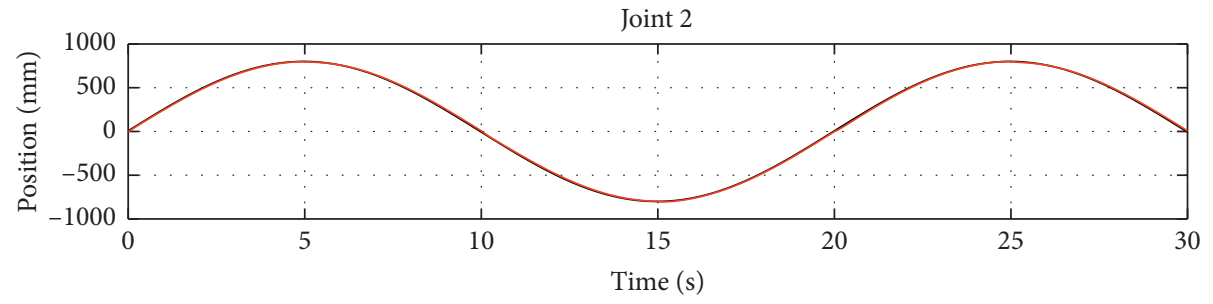

Given trajectory

Tracking trajectory

(b)

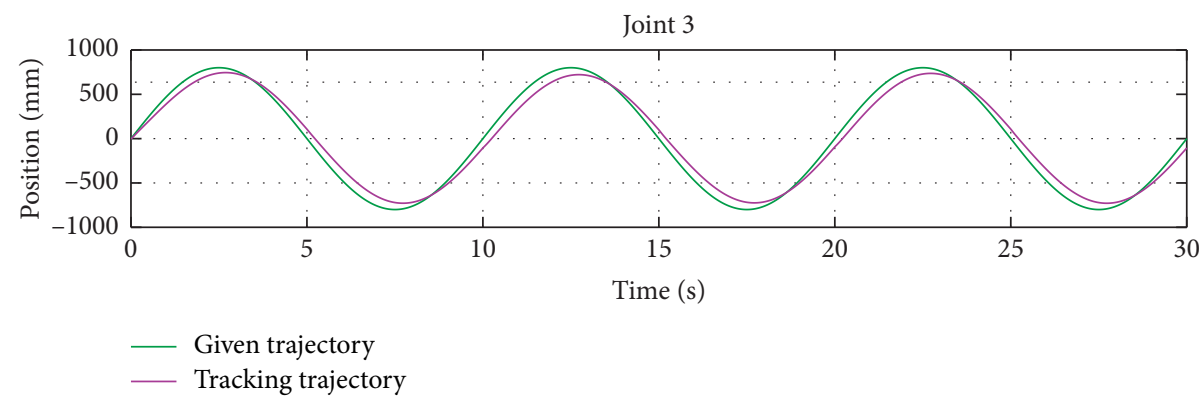

(c)

FIgURE 10: Trajectories of 3 joints without the LADRC strategy. (a) Trajectories of joint 1. (b) Trajectories of joint 2. (c) Trajectories of joint 3.

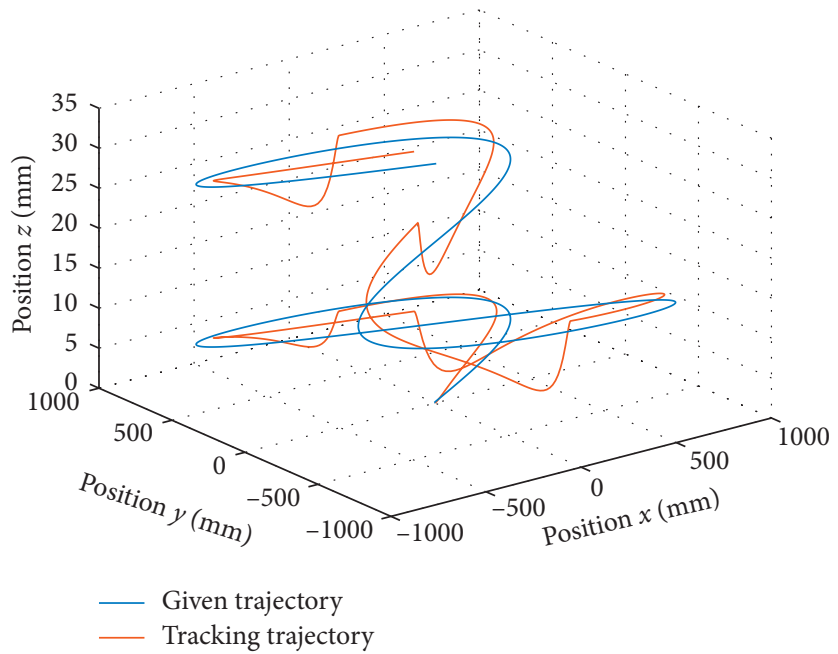

FIGURE 11: The tracking trajectory of without the LADRC strategy. 


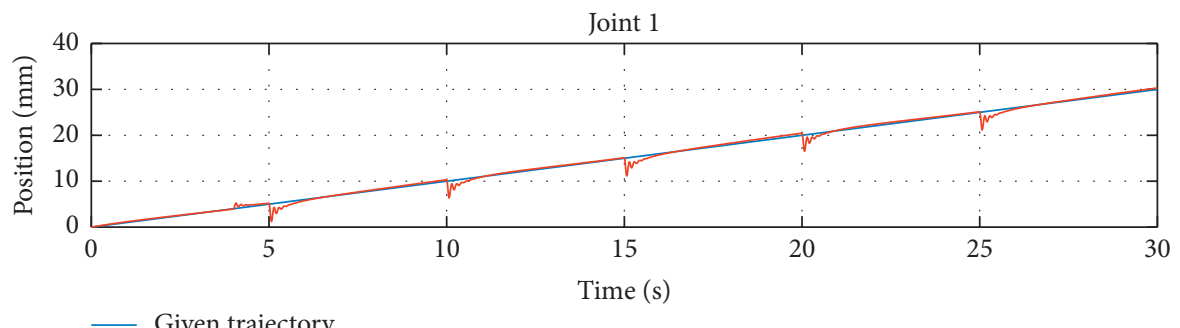

_ Tracking trajectory

(a)

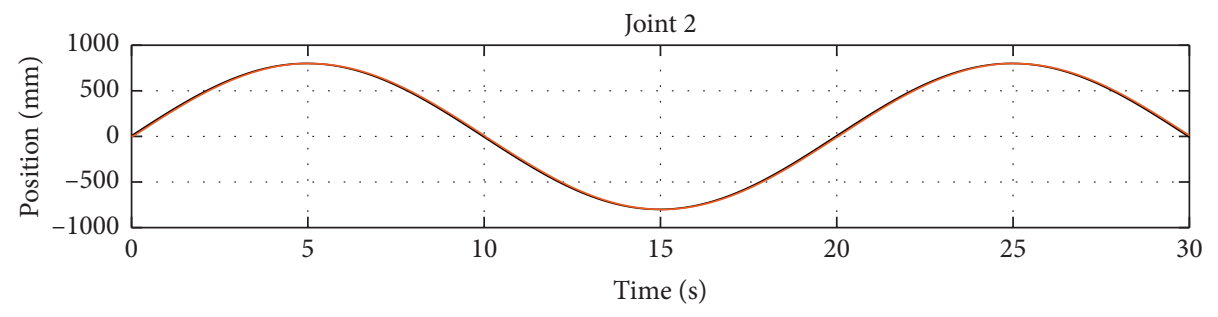

Given trajectory

Tracking trajectory

(b)

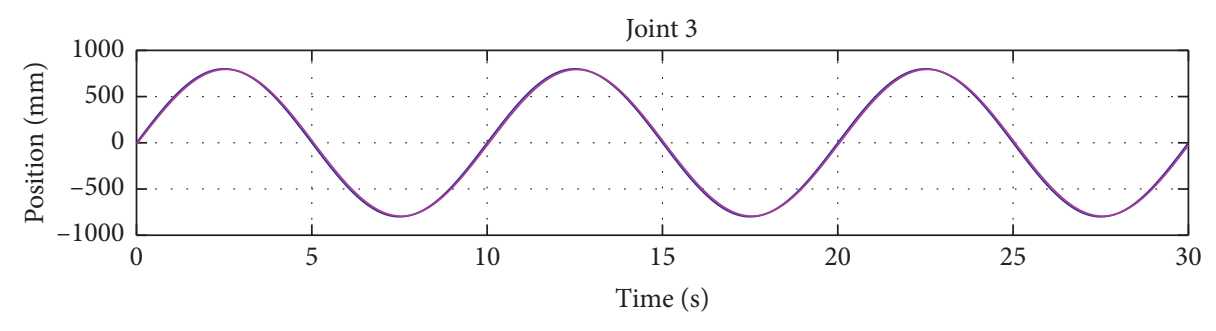

Given trajectory

Tracking trajectory

(c)

Figure 12: Trajectories of 3 joints with the LADRC strategy. (a) Trajectories of joint 1. (b) Trajectories of joint 2. (c) Trajectories of joint 3.

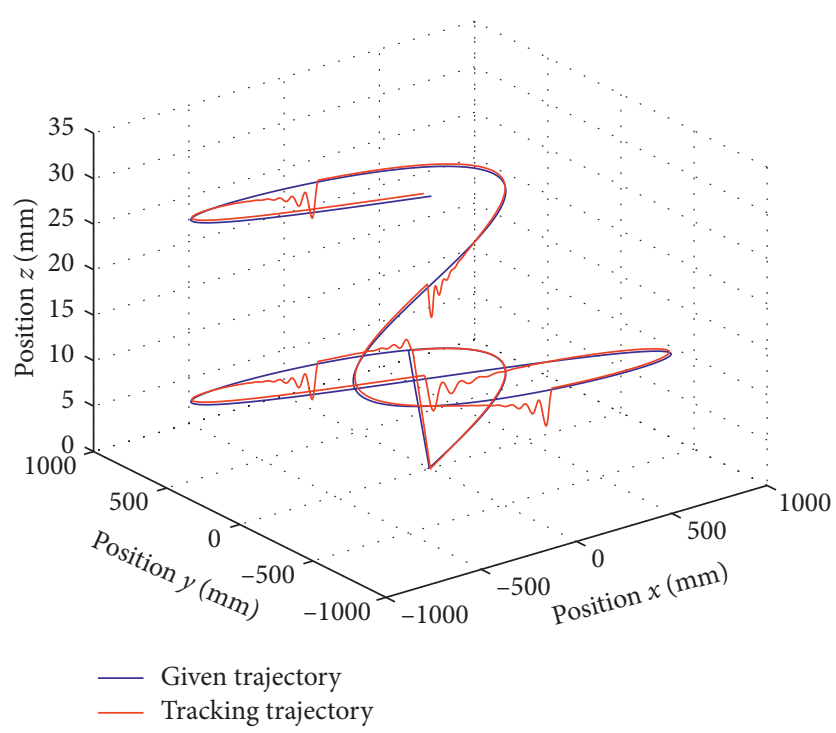

FIGURE 13: The tracking trajectory with the LADRC strategy. 


\section{Conclusion}

Aiming at the motion requirements, nonlinear characteristics, and the omission of the relevant factors for modeling Delta high-speed parallel robot, this paper proposes an LADRC strategy to be applied to the dynamic control of Delta high-speed parallel robot. Through the stability analysis, the LADRC strategy can realize the dynamic control of the trajectory tracking of Delta high-speed parallel robot, and when the robot is running at high speed, the stability of the model will not be affected by the uncertain factors of the system.

In order to verify the proposed LADRC strategy, the circular trajectory, a representative 8 -shaped space helical variance trajectory, and a triangular wave external disturbance with large amplitude are input, respectively. The PID method and the linear ADRC control strategy are used to simulate, respectively. The simulation and comparative analysis show that the LADRC strategy is applied to the Delta high-speed parallel robot control which has a good quick and precise real-time trajectory tracking and strong robustness.

\section{Data Availability}

The data used to support the findings of this study are available from the corresponding author upon request.

\section{Conflicts of Interest}

The authors declare that there are no conflicts of interest regarding the publication of this paper.

\section{Acknowledgments}

This work was supported by the Education Department of Jilin Province (Grant JJKH20200044KJ), the Jilin Provincial Development and Reform Commission (Grant 2018C0351), Jilin Provincial Science and Technology Department (Grants 20160101276JC and 20150312040ZG), and Project of Beihua University (Grant 201901012).

\section{References}

[1] Z. X. Cai, Fundamentals of Robotics, Mechanical Industry Press, Beijing, China, 2009.

[2] J. P. Merlet, Parallel Robots, Springer Press, Dordrecht, Netherlands, 2006.

[3] S. Cong and W. W. Shang, Parallel Robot: Modeling, Control Optimization and Application, Electronic Industry Press, Beijing, China, 2009.

[4] X. S. Chen, Z. L. Chen, and M. X. Kong, "The development and current situation of parallel robot research," Robot, vol. 24, no. 5, pp. 464-470, 2002.

[5] B. J. Yang, G. Q. Cai, J. M. Luo et al., "Research status of low DOF parallel robots," Machine Tool and Hydraulic, vol. 34, no. 5, pp. 202-205, 2006.

[6] Q. L. Ai, S. J. Zu, and F. Xu, "Research progress on kinematics and singularity of parallel mechanism," Journal of Zhejiang University: Engineering Edition, vol. 46, no. 8, pp. 1345-1359, 2012.
[7] U. Thakar, V. Joshi, and V. V. Yawahare, "Fractional-order PI controller design for PMSM: a model-based comparative study," in Proceedings of the International Conference on Automatic Control \& Dynamic Optimization Techniques, IEEE, Pune, India, September 2017.

[8] Q. Zhao, P. Wang, and J. Mei, "Controller parameter tuning of delta robot based on servo identification," Chinese Journal of Mechanical Engineering, vol. 28, no. 2, pp. 267-275, 2015.

[9] Y. Q. Wang and T. Huang, "Research on the parameter tuning method of Delta mechanism controller," Mechanical Design and Manufacturing, vol. 08, pp. 20-22, 2008.

[10] Y. Su, D. Sun, L. Ren, and J. K. Mills, "Integration of saturated PI synchronous control and PD feedback for control of parallel manipulators," IEEE Transactions on Robotics, vol. 22, no. 1, pp. 202-207, 2006.

[11] X.-G. Lu, M. Liu, and J.-X. Liu, "Design and optimization of interval type-2 fuzzy logic controller for delta parallel robot trajectory control," International Journal of Fuzzy Systems, vol. 19, no. 1, pp. 190-206, 2017.

[12] F. Pierrot, V. Nabat, O. Company, S. Krut, and P. Poignet, "Optimal design of a 4-DOF parallel manipulator: from academia to industry," IEEE Transactions on Robotics, vol. 25, no. 2, pp. 213-224, 2009.

[13] H. Z. Huang, Research on Structural Parameter Optimization and Motion Control of Delta Parallel Robot, Harbin Institute of Technology, Harbin, China, 2013.

[14] M. Rachedi, M. Bouri, and B. Hemici, "Application of an Ho control strategy to the parallel delta," in Proceedings of the 2012 2nd International Conference Communications, Computing and Control Applications (CCCA), December 2012.

[15] M. Rachedi, B. Hemici, and M. Bouri, "Application of the mixed sensitivity problem $\mathrm{H} \infty$ and $\mathrm{H} 2$ to the parallel delta," in Proceedings of the 3rd International Conference on Systems \& Control, October 2013.

[16] M. Rachedi, M. Bouri, and B. Hemici, "Hळ feedback control for parallel mechanism and application to delta robot," in Proceedings of the 22nd Mediterranean Conference on Control and Automation (MED), pp. 1476-1481, University of Palermo, Palermo, Italy: IEEE, June 2014.

[17] X. B. Guo, Kinematic Planning and Dynamic Control of Delta Parallel Robot, Technology of Guangdong University, Guangdong, China, 2015.

[18] G. Q. Gao, Q. Q. Ding, and W. Wang, "Application of RBF neural network sliding mode variable structure control in parallel robot," Industrial Instrumentation and Automation, vol. 02, pp. 35-39, 2012.

[19] K. Y. Bi, Research on the Control of a New Three Translation Parallel Robot Mechanism, Jiangsu University, Jiangsu, China, 2006.

[20] G. Q. Gao, Z. M. Fang, X. M. Niu et al., "Research on the smooth sliding mode control of a new three translation parallel robot mechanism," Modular Machine Tool and Automatic Machining Technology, vol. 11, pp. 81-83, 2004.

[21] C. Y. Lian, J. J. Zhang, L. H. Shi et al., "Joint simulation of 3DOF parallel robot based on fuzzy control," Machine Tool and Hydraulic, vol. 39, no. 09, pp. 122-125, 2011.

[22] O. Linda and M. Manic, "Uncertainty-Robust design of interval type-2 fuzzy logic controller for Delta parallel robot," IEEE Transactions on Industrial Informatics, vol. 7, no. 4, pp. 661-670, 2011.

[23] Y. C. Huang and Z. L. Huang, "Neural network based dynamic trajectory tracking of delta parallel robot," in Proceedings of International Conference on Mechatronics and Automation, pp. 1938-1941, Beijing, China: IEEE, 2015. 
[24] K. Zheng and C. Wang, Force/Position Hybrid Control of 6PUS-UPU Redundant Driven Parallel Manipulator Based on 2-DOF Internal Model Control, Hindawi Publishing Corp, London, UK, 2014.

[25] J. Z. Hui and Y. K. Yang, "Research on nonlinear dynamic control method of 2-DOF redundant drive parallel robot," Mechanical Engineering and Technology, vol. 5, no. 2, pp. 150-164, 2016.

[26] A. Vivas and P. Poignet, "Predictive functional control of a parallel robot," Control Engineering Practice, vol. 13, no. 7, pp. 863-874, 2005.

[27] M. A. Mirza, S. Li, and L. Jin, "Simultaneous learning and control of parallel stewart platforms with unknown parameters," Neurocomputing, vol. 266, pp. 114-122, 2017.

[28] J. K. Liu, MATLAB Simulation of Advanced PID Control, Electronic Industry Press, Beijing, China, 3rd edition, 2011.

[29] Y. C. Fan, Y. S. Li, Q. Yu et al., "Fuzzy adaptive PID control simulation of Stewart parallel robot based on ADAMS and MATLAB," Instruments and Equipment, vol. 3, no. 3, pp. 63-71, 2015.

[30] Mario Ramírez-NeriaHebertt et al., "Active disturbance rejection control applied to a Delta parallel robot in trajectory tracking tasks," Asian Journal of Control, vol. 17, no. 2, pp. 636-647, 2015.

[31] Z. Gao, "Scaling and bandwidth-parameterization based controller tuning," in Proceedings of the American Control Conference, pp. 4989-4997, Denver, CO, USA, June 2003.

[32] H. Y. Jin, L. L. Liu, and W. Y. Lan, "Stability conditions of linear ADRC for second order systems," Journal of Automation, vol. 44, no. 09, pp. 1725-1728, 2018.

[33] Z. Q. Chen, Y. Cheng, M. W. Sun et al., "Some progress of linear ADRC theory and engineering application," Information and Control, vol. 46, no. 03, pp. 257-266, 2017.

[34] D. Yuan, X. J. Ma, Q. H. Zeng et al., "Study on the frequency characteristics and parameter configuration of the linear ADRC for the second order system," Control Theory and Application, vol. 30, no. 12, pp. 1630-1640, 2013.

[35] R. Zhang, "Parameter identification based on series extended state observer," Journal of System Simulation, vol. 06, pp. 793-795, 2002.

[36] P. W. Li, Application of Advanced Control Theory in Control System of Cold Mill, Science and Technology of Beijing University, Beijing, China, 2019.

[37] B. Liu, J. Hong, and L. Wang, "Linear inverted pendulum control based on improved ADRC," Systems Science \& Control Engineering, vol. 7, no. 3, pp. 1-12, 2019.

[38] Y. Liu, H. Liu, and Y. Meng, "Active disturbance rejection control for a multiple-flexible-link manipulator," Journal of Harbin Institute of Technology, vol. 25, no. 1, pp. 18-28, 2018. 